\title{
Considerations in treating patients with advance lung cancer during the epidemic outbreak of novel coronavirus (SARS-CoV-2)
}

\author{
Yangyang Cai ${ }^{1}$ - Yinghui Xu ${ }^{1}$. Dongsheng Xu ${ }^{1}$. Yizhuo Wang ${ }^{1} \cdot$ Xu Wang $^{1}$. Chao Sun ${ }^{1} \cdot$ Ye Guo $^{1}$ - Shi Qiu ${ }^{1}$. \\ Kewei Ma' ${ }^{10}$
}

Received: 26 March 2020 / Accepted: 23 July 2020 / Published online: 3 August 2020

(c) Springer Science+Business Media, LLC, part of Springer Nature 2020

\begin{abstract}
The outbreak of pneumonia caused by novel coronavirus (SARS-CoV-2) in Wuhan, China, at the end of 2019 quickly escalated into a global health emergency. Since its outbreak until the 29th of April 2020, the pandemic has affected more than 3 million of people and caused 207,973 deaths globally. SARS-CoV-2 belongs to the $\beta$-coronavirus genus of the Coronavirus family, and it shares the same subfamily with severe acute respiratory syndrome-associated coronavirus (SARS-CoV) and Middle East respiratory syndrome-associated coronavirus (MERS-CoV), all of which lead to severe pneumonia. For cancer patients, especially those with lung cancers, their immune systems are compromised due to the disease itself as well as the treatment for cancer. The weakened immunity of these patients puts them at a higher risk of not only developing diseases but severe diseases. In this study, through a literature review and data collection, we focus on the selection and consideration of antitumor treatment strategies for advanced lung cancer during the coronavirus disease 2019 (COVID-19) epidemic.
\end{abstract}

Keywords COVID-19 $\cdot$ SARS-CoV-2 $\cdot$ Lung cancer $\cdot$ Coronavirus

\section{Introduction}

Coronaviruses belong to the genus Coronavirus of the family Coronaviridae in the order Nidovirales. They are viruses with single-stranded RNA enclosed within an envelope structure. Along with Middle East respiratory syndromeassociated coronavirus (MERS-CoV) and the severe acute respiratory syndrome-associated coronavirus (SARS-CoV), SARS-CoV-2 is the seventh coronavirus thus far that has been found to infect humans. Viral infections by MERSCoV, SARS-CoV and SARS-CoV-2 in human have high incidence rates of developing into acute respiratory distress syndrome (ARDS), for which the mortality rates are high $[1,2]$. The outbreak of SARS-CoV infections among humans between 2002 and 2003 led to a global epidemic that rapidly spread to more than 30 countries and killed approximately 800 people [1]. The mortality rate among all

Yangyang Cai and Yinghui Xu contributed equally to this work.

Kewei Ma

makw@jlu.edu.cn

1 Cancer Center, The First Hospital of Jilin University, 71 Xinmin Street, Changchun, 130021 Jilin, China patients infected with SARS-CoV was approximately $10 \%$. In 2012, a decade later, the first MERS-CoV infection was reported in Saudi Arabia [3]. The mortality rate for MERS$\mathrm{CoV}$ infection in the population was approximately $30 \%$ [2]. The transmission capacity of SARS-CoV-2 is considered relatively strong by the World Health Organization (WHO). During the novel coronavirus disease 2019 (COVID-19) outbreak, one population of patients who cannot be ignored is cancer patients. Based on a report by the Chinese Center for Disease Control and Prevention (CCDCP), in which the epidemiological characteristics of 72,314 COVID-19 cases (including confirmed cases and suspected positive cases) on mainland China as of February 11, 2020, were described and analyzed,107 $(0.5 \%)$ of the patients had preexisting cancers. Six of them died; therefore, the mortality rate was $5.6 \%$, higher than the overall mortality rate for the general population [4].

In the past 10 years, as the lives of cancer patients have extended due to improved treatment options, the number of cancer patients with compromised immune function has also expanded. Because respiratory viruses mainly attack lung tissues, patients with advanced lung cancers who already have impaired pulmonary function and a weakened immune system are more susceptible to SARS-CoV-2 infections, 
often with a poor prognosis [5]. Therefore, many of these cancer patients chose to suspend their treatment during the epidemic, not only to avoid possible infections from contact with the external environment but also to allow immune function to be properly restored so as to have some resistance to viral infections. However, because the duration of the epidemic could become extensive, long-term interruption of treatment will inevitably lead to tumor progression and an elevated risk of recurrence and metastasis. However, if a lung cancer patient is infected withSARS-CoV-2 but is only experiencing mild symptoms, how should the situation be handled appropriately to achieve effective control of the viral infection while preventing cancer recurrence in the lungs? Hence, proper management of the treatment for this special population of patients during the outbreak has become a top priority for oncologists. The current treatment for patients with advanced lung cancer consists of a comprehensive approach that includes chemotherapy, targeted therapy, and immunotherapy. In this article, after reviewing the literature and collecting relevant information on lung cancer treatment and viral infections, the advantages and disadvantages of treating advanced pulmonary tumors during the COVID-19 epidemic were analyzed from multiple different perspectives, with the aim of providing a theoretical basis for lung cancer treatment strategies during the outbreak.

\section{Chemotherapy}

Lung cancer has long been ranked as the malignant tumor with the highest incidence and mortality rate. Eighty-five percent of cancers in the lung are non-small cell lung cancer (NSCLC), and most NSCLC patients are already at advanced stage when seeking treatment $[6,7]$. As the most basic treatment for cancer treatment, chemotherapy has a broad scope of application and high intensity of action, and doctors have substantial experience using the treatment drugs. Chemotherapy is considered as the base treatment option for most advanced lung cancers without apparent driver genes. Cell cycle-specific drugs plus platinum-based reagents are the most frequent chemotherapeutic drug combinations. Once the drugs are administered, either through intravenous injection or oral consumption, they enter the body and act directly on the cell cycle to kill rapidly growing tumor cells. Many chemotherapeutic drugs are bone marrow inhibitors that inhibit blood cell production in the bone marrow; thus, they can lead to anemia and reductions in the numbers of platelets and absolute white blood cells and lymphocytes $[8,9]$. Increased risk for infections after chemotherapy has also been confirmed [10-12]. Additionally, patients may experience fatigue and adverse gastrointestinal reactions such as nausea and vomiting, which can cause difficulty in food intake and malnutrition. Such reactions can worsen a patient's situation and lead to further weakening of immunity, rendering patients undergoing chemotherapy as a susceptible population for SARS-CoV-2 infection. The first study ofSARS-CoV-2 infections in cancer patients by Liang et al. analyzed the detailed medical records (ending on January 31,2020 ) of 1590 COVID-19 patients and found that 18 patients (1\%) had a history of cancer, of whom 5 were lung cancer patients $(5 / 18,28 \%)$. Compared with patients with no cancer history, cancer patients exhibited a higher incidence of developing critical illness ( $38.9 \%$ vs. $7.9 \%, P=0.0003$ ). Moreover, patients who underwent chemotherapy/surgery in the past month $(6 / 14,42.9 \%)$ displayed an elevated risk for severe illness compared with those who did not receive treatment during the same period $(1 / 3,75.0 \%)$ [5]. Because of the already declined immune function in cancer patients, further chemotherapy-introduced damage to the functions of immune cells, such as granulocytes and lymphocytes, will not only raise patients' risk of COVID-19 but also increase the chance of developing critical illness once infected. Therefore, in regions of the COVID-19 outbreak where the viral load of SARS-CoV-2 are high and the risk of infection are greater, for patients whose cancer is in stable condition, delaying adjuvant chemotherapy to reduce the risk of viral infections is recommended. For lung cancer patients who have received chemotherapy and have SARS-CoV-2 infection, close attention should be paid to the condition of those patients, being aware of the risk of severe pneumonia. Timely interventions should be carried out to avoid irreversible damage. In areas where there is no significant outbreak of COVID-19 and the viral load and number of infected individuals is low and under control, chemotherapy should continue to be carried out at local clinics for patients with advanced lung cancer who meet the following criteria: (1) not infected with SARS-CoV-2; (2) in good health with all immunity markers falling within the normal range; and (3) well-tolerated current chemotherapy regimen. Special protective measures ought to be taken during treatment to minimize the chance of cross infections. For advanced lung cancer patients who have SARS-CoV-2 infection, regardless of whether the patients have mild symptoms or are asymptomatic carriers of the virus, the chemotherapy should be temporarily suspended to avoid the possibility of further progression of the viral infection because of the impaired patient immune function caused by chemotherapy treatment. Specific cancer treatment strategies should be developed as appropriate based on the clinical outcome of viral infections.

\section{Molecular targeted therapy}

Molecular targeted therapy is one of the breakthrough treatment options developed based on genetics. Because certain drugs have direct inhibitory effects on the pathway of 
cancer driver genes, they can directly block tumor growth and achieve better treatment outcomes while minimizing adverse reactions. In non-small-cell lung cancer (NSCLC), the most frequent mutation occurs in $E G F R$, with a rate of mutation of approximately $50 \%$, followed by $A L K$ fusion, with a positive rate of approximately $10 \%$. Some additional rare lung cancer driver genes include the fusion genes ROS1, $R E T, N T R K$ and $B R A F[13,14]$. Epidermal growth factor receptor (EGFR), a member of the ERBB family of tyrosine kinase receptors, is a transmembrane glycoprotein encoded by the p12 loci on chromosome 7. It forms a dimer with its ligand, which then binds the activated protein kinase to initiate the critical downstream signaling pathways (such as RAS-RAF-MEK-MAPK and PI3K-ALK-mTOR) that promote cell proliferation and angiogenesis and reduce metastasis and apoptosis [15]. Currently, the most frequently used drugs targeting EGFR in clinical practice are small molecule receptor tyrosine kinase inhibitors (TKIs). There are currently several types of EGFR-TKIs, including erlotinib, gefitinib, afatinib, and osimertinib. It was suggested in clinical studies that NSCLC patients treated with EGFR-TKIs showed an increased risk of interstitial lung disease (ILD), which is a prognostic indication for pulmonary fibrosis [16]. If a patient already exhibits detrimental signs of ILD after receiving EGFR-TKIs treatment and is then infected with SARS-CoV-2, the viral infection will undoubtedly aggravate the lung damage and likely lead to severe pneumonia. Therefore, it is necessary to immediately stop TKIs drug therapy and begin active anti-viral treatment in these patients. An appropriate treatment strategy for targeted therapy should be selected once treatment for the viral infection is completed.

It has been suggested that SARS-CoV-2 infects pulmonary cells through angiotensin-converting enzyme 2 (ACE2), a receptor that is abundantly expressed in type II alveolar epithelial cells (AT2 cells), kidney cells and gastrointestinal tract epithelial cells. AT2 cells are particularly vulnerable to viral infections $[17,18]$. AP2-related protein kinase 1 (AAK1) and cyclin G-related kinase (GAK) regulate receptor-mediated endocytosis and trans-Golgi network (TGN)transport [19-22]. Sunitinib and erlotinib are effective, non-selective inhibitors of AAK1 and GAK, respectively. A variety of viruses, such as Dengue virus (DENV) and Ebola virus, enter cells and produce infectious viral particles through AAK1- and GAK-mediated processes. In DENV- and Ebola-infected mice, the sunitinib-erlotinib combination treatment has prevented disease onset and reduced mortality [23]. An additional benefit of sunitinib and erlotinib treatment for patients is that it can alter the body's cytokine response (shifting the balance from pathogenesis to viral clearance) [24]. From that perspective, erlotinib may have an antiviral effect in treating COVID-19. If EGFR-TKI treatment was well tolerated by lung cancer patients and did not cause significant damage to pulmonary tissues, continued treatment with EGFR-TKIs under mild SARS-CoV-2 infections should still be an option, as long as the lungs do not display detectable changes in imaging features. However, changes in pulmonary tissues should be frequently monitored using imaging technologies.

In summary, we believe that patients with advanced lung cancer who do not have SARS-CoV-2 infections should continue their treatment with EGFR-TKIs. For lung cancer patients infected withSARS-CoV-2, if their lungs are fully functioning and without significant interstitial inflammation, the continued use of EGFR-TKIs is recommended, as these drugs may also have inhibitory effects on the viral infection. However, each patient's lungs should be closely monitored through imaging examinations during and after antiviral treatment to detect interstitial pneumonia. If the chest computed tomography (CT) of lung cancer patients with COVID-19shows apparent indications of interstitial changes, the use of EGFR-TKIs should be immediately suspended to prevent irreversible lung damage caused by the combinational effects of the drug and viral infection. However, no situationis absolutely static; thus, any treatment plan must be made based on each patient's specific situation.

\section{Immunotherapy}

Immunotherapy for lung cancer mainly refers to treatment through programmed death receptor 1 (PD-1)/programmed death receptor-ligand 1 (PD-L1). These monoclonal antibodies target programmed necrosis factors, removing the "brake" effect of tumors on the immune system and thereby rejuvenating the body's immunity to attack and kill tumor cells and suppress tumor growth. Xu et al. [25] performed the first pathological autopsy on a patient who died of COVID19. According to the report, the patient's lungs showed signs consistent with ARDS, including diffuse alveolar damage and pulmonary hyaline membrane formation, of which the overall pathological manifestations in the lungs were similar to those of SARS and MERS. When the researchers analyzed the peripheral blood using flow cytometry, they discovered that although the numbers of CD $4+$ and $\mathrm{CD} 8+\mathrm{T}$ cells in the blood were significantly reduced, the cells were in an over-activated state; i.e. Th17 cells were elevated, and $\mathrm{CD} 8+\mathrm{T}$ cells exhibited high cytotoxicity. These results suggest, to a certain extent, that the patient's immune system was severely damaged. Leukopenia and lymphocytopenia were both observed in the peripheral blood of patients in the acute phase of infection with SARS-CoV, MERS-CoV and SARS-CoV-2 [26-28]. The extent of T cell reduction was correlated with the severity of acute phase SARSCoV-2 infection in patients [28-30]. CD8 + cytotoxic T lymphocytes (CTLs) are the major immune cell type that clears respiratory viral infections. CTL deficiency in mice 
results in delayed clearance of viruses, while individuals with $\mathrm{T}$ cell deficiency associated with aging, immunosuppression, or cancer tend to have more severe infections and poor prognostic outcomes [31, 32]. However, the effects of killing invaded pathogens via the immune system can also simultaneously causes damage to normal cells in tissues [33, 34]. Therefore, the balance between immune protection and immunopathology is critical for host defense against respiratory viruses.

PD-1 and PD-L1 inhibitors, which are immune checkpoint inhibitors, are commonly used for lung cancer immunotherapy. Viral infection can lead to the increased expression of PD-1 and PD-L1 on the surface of antigen-specific T cells [35, 36], inhibit the proliferation of CD4 + and CD8 + T cells, reduce the secretion or spread of the cytokines interleukin-2 (IL-2) and interferon- $\gamma$ (IFN- $\gamma$ ), reduce the immune function of specific $\mathrm{T}$ lymphocytes, and even lead to organ failure. The above effects of viral infection can weaken the host's antiviral immune response, cause damage to target organs, and eventually lead to diseases associated with persistent viral infection. Alough blocking the PD-1-mediated signaling pathway can enhance the abilities of CD8 $+\mathrm{T}$ cells to proliferate, secrete cytokines and kill infected cells, thereby reducing the viral load theoretically [37-39], it will make activated CD8 $+\mathrm{T}$ cells further exhausted, and immune system will be damaged in another way. Patients who contract common respiratory viral infections while under immunotherapy may beat a higher risk for pneumonia [40], which is also very likely to progress into a critical condition due to a disrupted immune status. Hence, if a patient becomes infected with SARS-CoV-2, treatment with immune checkpoint inhibitors should be discontinued regardless of how mild or severe the symptoms may appear.

We believe that during the COVID-19 epidemic, advanced lung cancer patients who have not been infected with SARS-CoV-2 should continue immunotherapy treatment if their immune function is still normal. However, proper precautions and protection must be taken to avoid possible infections. In advanced lung cancer patients who have SARS-CoV-2 infection, taking into account of the impact of the viral infection on immune cells and the disrupted status of their immune system, suspending treatment with immune checkpoint inhibitors is an appropriate consideration. The patient should first undergo antiviral treatment to control the infection; immunotherapy can then be resumed as appropriate.

\section{Conclusions}

In summary, during the COVID-19 epidemic, cancer patients, especially lung cancer patients, represent one of the focal points of epidemic prevention due to their weakened immunity, severe symptoms and high fatality rate. When lung cancer patients who are receiving anticancer therapies exhibit symptoms such as fever or cough, suggesting possible infection, COVID-19 differential diagnosis should be carefully performed, and the risk of COVID-19 should be assessed for appropriate early intervention. For patients with advanced lung cancer who have contracted SARS-CoV-2, continued chemotherapy and immunotherapy can generate a greater risk of progressing into severe viral infection. Therefore, we recommend temporarily suspending the use of drugs; however, for targeted therapies such as EGFRTKIs, decisions should be made based on the presence or absence of lung damage. Briefly, in clinical settings, situations always change, and each case requires careful review to make decisions based on the specific status of each patient.

Acknowledgements None

Funding Yinghui Xu was supported by Youth Foundation of The First Hospital of Jilin University (Grant ID: JDYY82017020). Yinghui Xu was also supported by Xisike Clinical Oncology Research Foundation (CSCO-Haosen) (Grant ID: Y-HS2017-062). This research received no specific grant from any funding agency in the public, commercial, or not-for-profit sectors.

\section{Compliance with ethical standards}

Conflict of interest The authors declare no conflict of interest.

\section{References}

1. Peiris JSM, Guan Y, Yuen KY. Severe acute respiratory syndrome. Nat Med [Internet]. 2004 [cited 2020 Feb 25];10:S88-97. Available from: https://www.nature.com/articles/nm1143

2. World Health Organization. Middle East respiratory syndrome coronavirus (MERS-CoV)—update. World Health Organization, Geneva, Switzerland [Internet]. 2014. Available from: https:// www.who.int/csr/don/2014_03_25/en/

3. Zaki AM, van Boheemen S, Bestebroer TM, Osterhaus ADME, Fouchier RAM. Isolation of a Novel Coronavirus from a Man with Pneumonia in Saudi Arabia. N Engl J Med [Internet]. 2012 [cited 2020 Feb 25];367:1814-20. Available from: https://www.nejm. org/doi/abs/10.1056/NEJMoa1211721

4. Zhonghua Liu Xing Bing Xue Za Zhi Novel Coronavirus Pneumonia Emergency Response Epidemiology Team 2020 The epidemiological characteristics of an outbreak of 2019 novel coronavirus diseases (COVID-19) in China 41145151

5. Liang W, Guan W, Chen R, Wang W, Li J, Xu K, et al. Cancer patients in SARS-CoV-2 infection: a nationwide analysis in China. The Lancet Oncology [Internet]. 2020 [cited 2020 Feb 26];S1470204520300966. Available from: https://linkinghub .elsevier.com/retrieve/pii/S1470204520300966

6. Cao M, Chen W. Epidemiology of lung cancer in China. Thorac Cancer. 2019;10:3-7.

7. Zappa C, Mousa SA. Non-small cell lung cancer: current treatment and future advances. Transl Lung Cancer Res. 2016;5:288-300.

8. Kuhn JG. Chemotherapy-associated hematopoietic toxicity. Am J Health Syst Pharm. 2002;59:S4-7. 
9. Schiller JH, Harrington D, Belani CP, Langer C, Sandler A, Krook $\mathrm{J}$, et al. Comparison of four chemotherapy regimens for advanced non-small-cell lung cancer. N Engl J Med. 2002;346:92-8.

10. Nordvig J, Aagaard T, Daugaard G, Brown P, Sengeløv H, Lundgren J, et al. Febrile Neutropenia and Long-term Risk of Infection Among Patients Treated With Chemotherapy for Malignant Diseases. Open Forum Infect Dis. 2018;5:ofy255.

11. Lyman GH, Michels SL, Reynolds MW, Barron R, Tomic KS, Yu J. Risk of mortality in patients with cancer who experience febrile neutropenia. Cancer. 2010;116:5555-63.

12. Whittle SB, Williamson KC, Russell HV. Incidence and risk factors of bacterial and fungal infection during induction chemotherapy for high-risk neuroblastoma. Pediatr Hematol Oncol. 2017;34:331-42

13. Gazdar AF. EGFR mutations in lung cancer: different frequencies for different folks. J Thorac Oncol. 2014;9:139-40.

14. Devarakonda S, Morgensztern D, Govindan R. Genomic alterations in lung adenocarcinoma. Lancet Oncol. 2015;16:e342-351.

15. Scagliotti GV, Selvaggi G, Novello S, Hirsch FR. The biology of epidermal growth factor receptor in lung cancer. Clin Cancer Res. $4227 \mathrm{~s} ; 10: 4227 \mathrm{~s}-\mathrm{s} 42324232$.

16. Kato T, Nishio K. Clinical aspects of epidermal growth factor receptor inhibitors: benefit and risk. Respirology. 2006;11:693-8.

17. Zou X, Chen K, Zou J, Han P, Hao J, Han Z. The single-cell RNA-seq data analysis on the receptor ACE2 expression reveals the potential risk of different human organs vulnerable to Wuhan 2019-nCoV infection. Front Med. [Epub ahead of print].

18. Zhao Y, Zhao Z, Wang Y, Zhou Y, Ma Y, Zuo W. Single-cell RNA expression profiling of ACE2, the putative receptor of Wuhan 2019-nCov [Internet]. Bioinformatics; 2020 Jan. Available from: https://biorxiv.org/lookup/doi/10.1101/2020.01.26.919985

19. Olusanya O, Andrews PD, Swedlow JR, Smythe E. Phosphorylation of threonine 156 of the $\mu 2$ subunit of the AP2 complex is essential for endocytosis in vitro and in vivo. Current Biology [Internet]. 2001 [cited $2020 \mathrm{Feb} 27$ ];11:896-900. Available from: https://linkinghub.elsevier.com/retrieve/pii/S0960982201002408

20. Ricotta D, Conner SD, Schmid SL, von Figura K, Höning S. Phosphorylation of the AP2 $\mu$ subunit by AAK1 mediates high affinity binding to membrane protein sorting signals. The Journal of Cell Biology [Internet]. 2002 [cited 2020 Feb 27];156:791-5. Available from: https://rupress.org/jcb/article/156/5/791/32369/Phosp horylation-of-the-AP2-\%CE\%BC-subunit-by-AAK1

21. Umeda A. Identification of the universal cofactor (auxilin 2) in clathrin coat dissociation. European Journal of Cell Biology [Internet]. 2000 [cited $2020 \mathrm{Feb} 27$ ]; 79:336-42. Available from: https://linkinghub.elsevier.com/retrieve/pii/S0171933504700370

22. Zhang CX, Engqvist-Goldstein ÅY, Carreno S, Owen DJ, Smythe E, Drubin DG. Multiple Roles for Cyclin G-Associated Kinase in Clathrin-Mediated Sorting Events: GAK in ClathrinMediated Trafficking. Traffic [Internet]. 2005 [cited $2020 \mathrm{Feb}$ 27];6:1103-13. Available from: https://doi.wiley.com/10.111 1/j.1600-0854.2005.00346.x

23. Bekerman E, Neveu G, Shulla A, Brannan J, Pu S-Y, Wang S, et al. Anticancer kinase inhibitors impair intracellular viral trafficking and exert broad-spectrum antiviral effects. J Clin Invest. 2017;127:1338-522.

24. Pu S-Y, Xiao F, Schor S, Bekerman E, Zanini F, Barouch-Bentov R, et al. Feasibility and biological rationale of repurposing sunitinib and erlotinib for dengue treatment. Antiviral Res. 2018;155:67-75.

25. Xu Z, Shi L, Wang Y, Zhang J, Huang L, Zhang C, et al. Pathological findings of COVID-19 associated with acute respiratory distress syndrome. Lancet Respir Med. 2020;
26. Assiri A, Al-Tawfiq JA, Al-Rabeeah AA, Al-Rabiah FA, Al-Hajjar S, Al-Barrak A, et al. Epidemiological, demographic, and clinical characteristics of 47 cases of Middle East respiratory syndrome coronavirus disease from Saudi Arabia: a descriptive study. Lancet Infect Dis. 2013;13:752-61.

27. Wong RSM, Wu A, To KF, Lee N, Lam CWK, Wong CK, et al. Haematological manifestations in patients with severe acute respiratory syndrome: retrospective analysis. BMJ. 2003;326:1358-62.

28. Zhang J-J, Dong X, Cao Y-Y, Yuan Y-D, Yang Y-B, Yan Y-Q, et al. Clinical characteristics of 140 patients infected by SARSCoV-2 in Wuhan, China. Allergy. 2020;

29. Li T, Qiu Z, Zhang L, Han Y, He W, Liu Z, et al. Significant changes of peripheral $\mathrm{T}$ lymphocyte subsets in patients with severe acute respiratory syndrome. J Infect Dis. 2004;189:648-51.

30. Li T, Qiu Z, Han Y, Wang Z, Fan H, Lu W, et al. Rapid loss of both CD4+ and CD8+ T lymphocyte subsets during the acute phase of severe acute respiratory syndrome. Chin Med J. 2003;116:985-7.

31. Widmer K, Zhu Y, Williams JV, Griffin MR, Edwards KM, Talbot HK. Rates of Hospitalizations for Respiratory Syncytial Virus, Human Metapneumovirus, and Influenza Virus in Older Adults. The Journal of Infectious Diseases [Internet]. 2012 [cited 2020 Feb 27];206:56-62. Available from: https://academic.oup.com/ jid/article-lookup/doi/10.1093/infdis/jis309

32. Matias G, Taylor R, Haguinet F, Schuck-Paim C, Lustig R, Shinde V. Estimates of hospitalization attributable to influenza and RSV in the US during 1997-2009, by age and risk status. BMC Public Health [Internet]. 2017 [cited 2020 Feb 27]; 17:271. Available from: https://bmcpublichealth.biomedcentral.com/artic les/10.1186/s12889-017-4177-z

33. Cannon MJ, Openshaw PJ, Askonas BA. Cytotoxic T cells clear virus but augment lung pathology in mice infected with respiratory syncytial virus. J Exp Med. 1988;168:1163-8.

34. Alwan WH, Kozlowska WJ, Openshaw PJ. Distinct types of lung disease caused by functional subsets of antiviral T cells. J Exp Med. 1994;179:81-9.

35. Keir ME, Butte MJ, Freeman GJ, Sharpe AH. PD-1 and its ligands in tolerance and immunity. Annu Rev Immunol. 2008;26:677-704.

36. Wei F, Zhong S, Ma Z, Kong H, Medvec A, Ahmed R, et al. Strength of PD-1 signaling differentially affects T-cell effector functions. Proc Natl Acad Sci USA. 2013;110:E2480-2489.

37. Im SJ, Hashimoto M, Gerner MY, Lee J, Kissick HT, Burger MC, et al. Defining CD8+ T cells that provide the proliferative burst after PD-1 therapy. Nature. 2016;537:417-21.

38. McNally B, Ye F, Willette M, Flaño E. Local blockade of epithelial PDL-1 in the airways enhances T cell function and viral clearance during influenza virus infection. J Virol. 2013;87:12916-24.

39. Erickson JJ, Gilchuk P, Hastings AK, Tollefson SJ, Johnson M, Downing $\mathrm{MB}$, et al. Viral acute lower respiratory infections impair CD8+ T cells through PD-1. J Clin Invest. 2012;122:2967-82.

40. Topalian SL, Hodi FS, Brahmer JR, Gettinger SN, Smith DC, McDermott DF, et al. Safety, Activity, and Immune Correlates of Anti-PD-1 Antibody in Cancer. N Engl J Med [Internet]. 2012 [cited 2020 Feb 27];366:2443-54. Available from: https://www. nejm.org/doi/10.1056/NEJMoa1200690

Publisher's Note Springer Nature remains neutral with regard to jurisdictional claims in published maps and institutional affiliations. 